\title{
Characterization of the PTW 34031 ionization chamber (PMI) at RCNP with high energy neutrons ranging from $100-392 \mathrm{MeV}$
}

\author{
C. Theis ${ }^{1 \mathrm{a}}$, P. Carbonez, E. Feldbaumer, D. Forkel-Wirth, L. Jaegerhofer, M. Pangallo, D. Perrin, C. Urscheler, S. Roesler and H. \\ Vincke, M. Widorski ${ }^{1}$

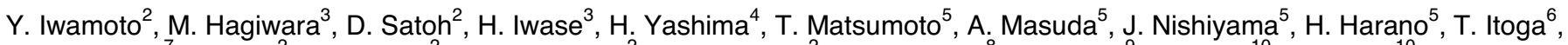 \\ T. Nakamura ${ }^{7}$, T. Sato ${ }^{2}$, Y. Nakane ${ }^{2}$, H. Nakashima ${ }^{2}$, Y. Sakamoto ${ }^{2}$, S. Taniguchi ${ }^{8}$, N. Nakao ${ }^{9}$, A. Tamii $^{10}$, T. Shima ${ }^{10}$, K. \\ Hatanaka $^{10}$
}

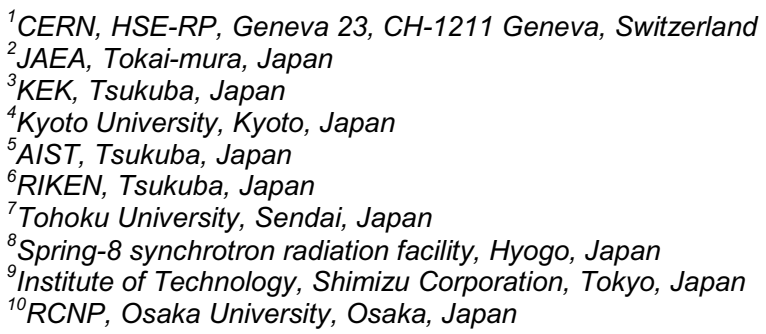

\begin{abstract}
Radiation monitoring at high energy proton accelerators poses a considerable challenge due to the complexity of the encountered stray radiation fields. These environments comprise a wide variety of different particle types and span from fractions of electron-volts up to several terra electron-volts. As a consequence the use of Monte Carlo simulation programs like FLUKA is indispensable to obtain appropriate field-specific calibration factors. At many locations of the LHC a large contribution to the particle fluence is expected to originate from high-energy neutrons and thus, benchmark experiments with mono-energetic neutron beams are of high importance to verify the aforementioned detector response calculations. This paper summarizes the results of a series of benchmark experiments with quasi mono-energetic neutrons of 100,140,200, 250 and $392 \mathrm{MeV}$ that have been carried out at RCNP - Osaka University, during several campaigns between 2006 and 2014.
\end{abstract}

\footnotetext{
${ }^{\text {a }}$ Corresponding author: christian.theis@cern.ch
} 


\section{Introduction}

Radiation monitoring at high-energy proton accelerators poses a considerable challenge due to the complexity of the encountered stray radiation fields. On one hand they span over an energy range comprising energies from fractions of electron-volts $(\mathrm{eV})$ up to several $\mathrm{TeV}$. On the other hand also the composition of the fields is very complex as it comprises many different particle types (hadrons, leptons, photons). At CERN gas-filled ionization chambers are commonly used in such mixed fields as they are sensitive to many different particle types which directly or indirectly cause ionization of the active detection medium. However, standard calibration sources like ${ }^{137} \mathrm{Cs},{ }^{60} \mathrm{Co}, \mathrm{Am}-\mathrm{Be}$ and $\mathrm{Pu}-\mathrm{Be}$ neither cover the full energy range nor the mixed particle composition of the radiation environments in which these detectors are eventually utilized. As a consequence the use of Monte Carlo simulation programs like FLUKA (1)(2) is indispensable to study and obtain an appropriate monitor calibration depending on the radiation field (3). As shown in Ref. (4) calibration-source based calibration can be applicable behind thick lateral shielding but this depends on the detector as well as the actual radiation field. In any case a thorough characterization and understanding of the detector response to mixed radiation fields is of high importance.

At CERN an air-filled ionization chamber under atmospheric pressure PTW-34031, which internally is named PMI and manufactured by PTW, is utilized for the measurement of dose in the LHC experiment caverns during the operation of the machine as well as the determination of the residual dose after the beam has been turned off. The response of this monitor to mixed fields has been studied with the help of Monte Carlo simulations as well as experimental benchmarks (5). The results generally confirmed good agreement of the FLUKA calculations and the experiments but due to the fact that the detector yields integral results, independent of particle species and energy, no detailed benchmark-based conclusion could be drawn for individual particle types.

In the radiation fields present during accelerator operation at CERN, a large contribution to the particle fluence is expected to originate from high-energy neutrons. Hence, benchmark experiments with mono-energetic neutron beams are indispensable to verify the highly important neutron part of the aforementioned detector response calculations. Around the LHC a significant contribution of neutrons with energies above $100 \mathrm{MeV}$ is to be expected and thus, a series of benchmark experiments with quasi mono- energetic neutrons of 100,140, 200, 250 and $392 \mathrm{MeV}$ have been carried out during several campaigns between 2006 and 2014. This paper includes the results of the latest experiment conducted at the end of 2014 as well as an overall summary of the whole campaign.

\section{Benchmark experiments at RCNP}

\subsection{Setup}

The Research Center for Nuclear Physics (RCNP) at the University of Osaka houses a unique chain of cyclotrons which is used mainly for Quantum Chromodynamics (QCD) studies. An AVF cyclotron is acting as an injector to a ring cyclotron which can deliver proton beams of up to $400 \mathrm{MeV}$ to various experimental halls (6) as depicted in Figure 1. Among these facilities there is a time of flight (TOF) tunnel of $100 \mathrm{~m}$ length which is available for experiments with quasi-mono-energetic neutrons. In our experiment protons of $100,140,200,250$ and $392 \mathrm{MeV}$ were directed at a $1 \mathrm{~cm}$ thick ${ }^{7} \mathrm{Li}$ target to produce quasi mono-energetic neutrons at the respective energies. All charged particles were deflected by a beam swinger magnet. The remaining beam of neutral particles is collimated by an iron collimator of $1.5 \mathrm{~m}$ with an opening of $10 \mathrm{~cm}$ (horizontally) x $12 \mathrm{~cm}$ (vertically) before entering the TOF tunnel.

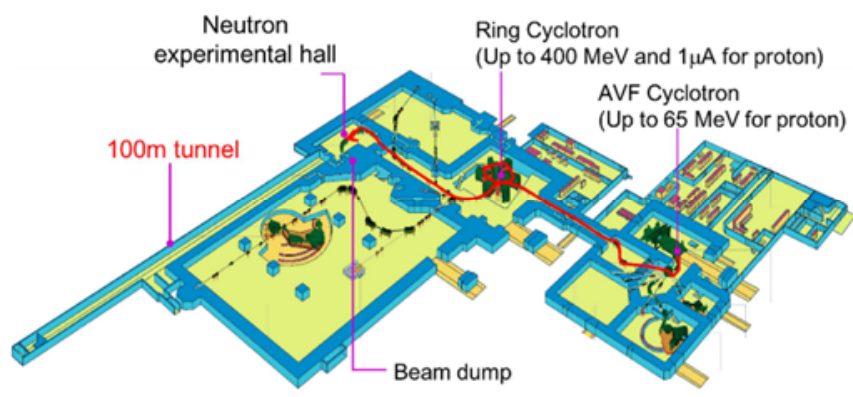

Figure 1: Schematic of the layout of the RCNP facility.

For the experiment two identical detectors were used which allowed for checking the equipment for any kind of malfunction. As illustrated in Figure 2 the monitors were placed on in the TOF tunnel at a certain distance from the target during the irradiation. This distance was determined by the prerequisite that the whole detector should be covered homogeneously by the neutron beam which, coming from the target, is divergent. Taking the beam extensions at the end of the collimator and the detector geometry as well as the dimensions into account it was found that for the PMI a distance of at least $18 \mathrm{~m}$ with respect to the target safely fulfills this requirement. With exception of 2014 the detectors were always placed in a distance of $25 \mathrm{~m}$ due to the fact that the space in front was occupied by other material and equipment that was tested during the same campaigns. In 2014 the chambers were moved closer and located at a distance of $18 \mathrm{~m}$ as the space behind our detectors was made available for parasitic irradiation tests of electronics. In order to compare the results all values have been scaled and normalized to the same distance of $18 \mathrm{~m}$ using the $1 / \mathrm{r}^{2}$ law. 


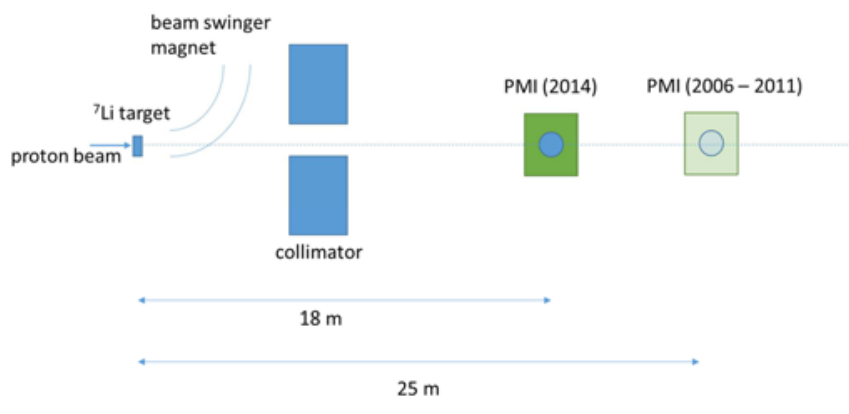

Figure 2: Sketch of the experimental setup at RCNP used in the years $2006-2011$, when the PMI was located in a distance of $25 \mathrm{~m}$ from the target, as well as 2014 when the detector was placed closer to allow for parasitic measurements behind it.

\subsection{Simulation of the experiment using quasi-monoenergetic neutrons from $100 \mathrm{MeV}$ up to $392 \mathrm{MeV}$ peak energy}

In order to be as accurate as possible the actual neutron fluence spectrum should be used as a source term in the calculations instead of mono-energetic neutrons. Over the years a complete set of measurements were conducted covering the energy range from 100 up to $392 \mathrm{MeV}$. For each of those scenarios the neutron fluence spectra have been determined by Iwamoto et al. (7)(8) (see Figure 3) and were used in the subsequent simulation of the experiment to characterize the PMI's response.

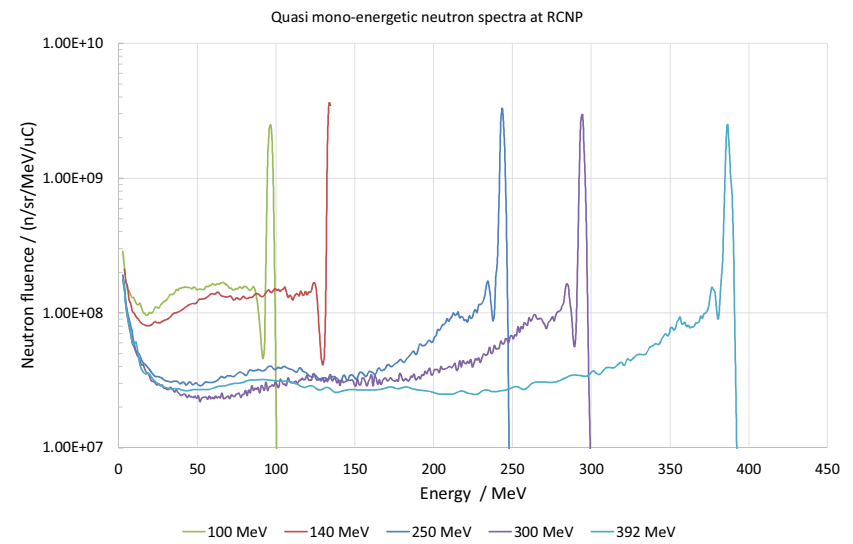

Figure 3: Neutron fluence spectrum per primary proton in lethargy representation, originating from protons with an energy of 100 up to $392 \mathrm{MeV}$ impinging on a $1 \mathrm{~cm}$ thick ${ }^{7} \mathrm{Li}$ target. The spectra were measured at RCNP Osaka between 2009 and 2014, using an organic NE213 liquid scintillator (7)(8).

For this purpose the detector's response function to neutrons was convoluted with the measured neutron spectrum and the resulting detector current was normalized to $1 \mathrm{uA}$ proton current on the Lithium target. These results were subsequently compared to the measured values.

\section{Comparison of simulation and measurements}

First measurements were conducted in 2006 with neutrons of
250 and $392 \mathrm{MeV}$ peak energy. First comparisons to simulations, which used neutron spectra that had been determined prior to these measurements, yielded a discrepancy that was not completely understood. Therefore, these measurements were repeated in 2009 and the respective neutron spectra were measured during the same campaign by Iwamoto et al. (7). During the second experiment the measurements of the first tests could be reproduced and verified. Yet, the discrepancy with respect to the simulations remained. At $250 \mathrm{MeV}$ an underestimation of the calculations of about $15 \%$ was found whereas at 392 $\mathrm{MeV}$ an underestimation of even $38 \%$ could be observed.

One has to keep in mind that during the $(p, n)$ reaction of protons impinging on a ${ }^{7} \mathrm{Li}$ target not only neutrons but also other particles are emitted. All charged particles are deflected by the beam swinger magnet but a photon component remains. The spectra measurements that were performed with the NE213 scintillator discriminated the photon component, but for ionization chambers this is not possible. First assumptions that the contribution might generally be negligibly small were proven false, as the measured charge for the neutron energy of $392 \mathrm{MeV}$ was significantly higher than the values obtained from simulations which assumed a pure neutron beam. Unfortunately the neutron TOF facility at RCNP has not been characterized with respect to photons and thus, no detailed information about the photon spectra was available. Therefore, it was attempted to calculate the respective spectra using FLUKA simulations of the proton interaction with the ${ }^{7} \mathrm{Li}$ target and the results are shown in Figure 4.

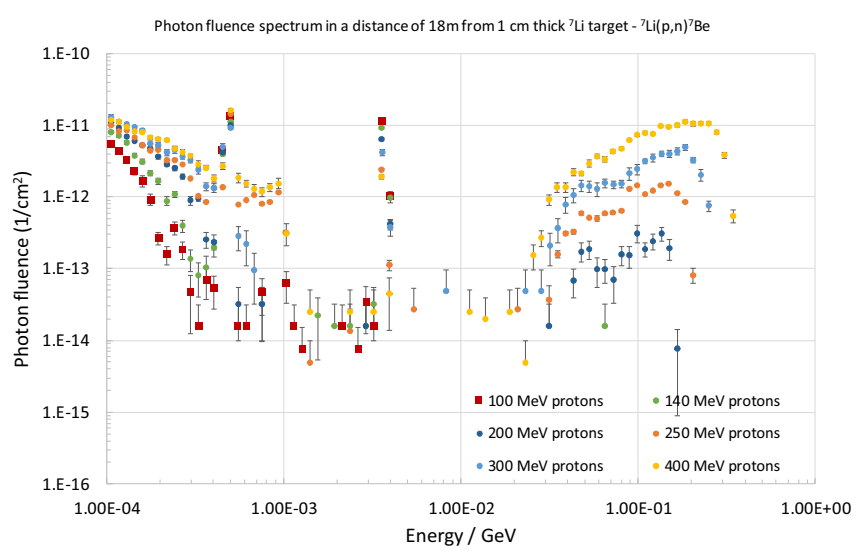

Figure 4: Photon fluence spectra per primary particle obtained from FLUKA calculations of protons with energies ranging between 100 and $392 \mathrm{MeV}$ impinging on a $1 \mathrm{~cm}$ thick ${ }^{7} \mathrm{Li}$ target.

Figure 4 nicely illustrates the distinction between low-energetic photons originating from the de-excitation of ${ }^{7} \mathrm{Be}$ as well as a notable high-energy component above 10 $\mathrm{MeV}$.

On one hand these findings corroborated the hypothesis that there is a non-negligible photon component which needs to be taken into account when using ionization chambers in the field at RCNP. On the other hand it also triggered a more detailed investigation of the simulation of the ${ }^{7} \mathrm{Li}$ target reaction using FLUKA. As discussed in detail in (3) the 
process is quite intricate to model as there is a low-energy photon component $(<1 \mathrm{MeV})$ which originates from excited states of ${ }^{7} \mathrm{Be}$ as well as a high-energy contribution that can be attributed to the decay of $\pi^{0}$ produced according to inelastic nucleon-nucleon models (9). The energy threshold for pion production is at $290 \mathrm{MeV}$ for a fixed target configuration and for incident proton energies of $392 \mathrm{MeV}$ this threshold is easily overcome and reliable result are obtained. At proton energies of $250 \mathrm{MeV}$ which is below the production threshold of pions one would not expect their creation and thus, no occurrence of high-energy photons due to $\pi^{0}$ decay. However, one has to take into account that nucleons are not at rest but subject to Fermi motion which in turn allows for pion production even at energies below 290 $\mathrm{MeV}$. It should be kept in mind that in FLUKA the radial dependence of the Fermi motion is implemented using the approximated shell model and density distributions. Consequently, these simplifications may have a notable impact on the obtained pion production for ${ }^{7} \mathrm{Li}$ and incident proton energies of $250 \mathrm{MeV}$.

There is also some uncertainty regarding the accuracy of the prediction of the low-energetic photons $(<1 \mathrm{MeV})$ due to the use of a so-called optical potential to model scattering processes in Lithium. As the nuclear levels are widely spaced the statistical assumptions of the models can be too coarse for detailed simulations (10). However, as shown in (3) for the $250 \mathrm{MeV}$ scenario $67 \%$ of the photon response of the PMI can be attributed to high energetic gammas $>1$ $\mathrm{MeV}$ and this value even rises to $93 \%$ considering $392 \mathrm{MeV}$ protons impinging on the target. In the latter case the influence of the approximations due to the Fermi motion are expected to be of lower importance as the primary energy of the incident protons is clearly beyond the pion production threshold, whereas this is not the case for the $250 \mathrm{MeV}$ scenario.

Looking once more at Figure 4 it can be seen that the high-energy photon fluence starts to significantly diminish with decreasing energy of the primary protons and at 140 $\mathrm{MeV}$ and below it has completely vanished due to the absence of the $\pi^{0}$ decay. It was found that for primary proton energies of $200 \mathrm{MeV}$ and below the photon contribution of the ${ }^{7} \mathrm{Li}$ target reaction to the simulated detector response is below $1 \%$ (11). Thus, it can be considered as negligible whereas at energies of $250 \mathrm{MeV}$ or higher their contribution cannot be neglected (3) and must be taken into account. A detailed summary of all measured as well as simulated results can be found in Table 1 .

Table 1 Summary of the measured as well as simulated detector response normalized per unit proton current on the Lithium target (3)(11). The simulations also include explicitly the photon contribution based on the spectra shown in Figure 4. It should be noted that all values have been scaled to reflect a setup at the same distance of $18 \mathrm{~m}$ from the target, whereas in 2009 and 2011 measurements had to be conducted at $25 \mathrm{~m}$. The uncertainties include statistical fluctuations of the measurements \& simulations as well as a systematic error of $10 \%$ for the beam intensity monitoring as well as an overall uncertainty of $5 \%$ for the neutron fluence measurements.

\begin{tabular}{|c|c|c|c|}
\hline $\begin{array}{c}\text { Energy } \\
(\mathrm{MeV})\end{array}$ & $\begin{array}{c}\text { Measured } \\
\text { current per }\end{array}$ & $\begin{array}{c}\text { Calculated } \\
\text { current per beam }\end{array}$ & $\begin{array}{c}\text { Calculated current } \\
\text { per beam intensity }\end{array}$ \\
\hline
\end{tabular}

\begin{tabular}{|c|c|c|c|}
\hline & $\begin{array}{c}\text { beam } \\
\text { intensity } \\
(\mathbf{p A} / \mu \mathbf{A})\end{array}$ & $\begin{array}{c}\text { intensity }(\mathbf{p A} / \boldsymbol{\mu} \mathbf{A}) \\
(\text { neutrons only) }\end{array}$ & $\begin{array}{c}(\mathbf{p A} / \mu \mathbf{A}) \\
(\text { neutrons }+\gamma)\end{array}$ \\
\hline $\mathbf{1 0 0}$ & $40.8+/-15 \%$ & $33.4+/-5 \%$ & $33.4+/-5 \%$ \\
\hline $\mathbf{1 4 0}$ & $36.3+/-10 \%$ & $35.1+/-9 \%$ & $33.4+/-5 \%$ \\
\hline $\mathbf{2 0 0}$ & $30.1+/-11 \%$ & $29.7+/-11 \%$ & $29.7+/-11 \%$ \\
\hline $\mathbf{2 5 0}$ & $28.9+/-3 \%$ & $24.5+/-7 \%$ & $30.9+/-7 \%$ \\
\hline $\mathbf{3 0 0}$ & $28.6+/-12 \%$ & $24.6+/-5 \%$ & $36.5+/-6 \%$ \\
\hline $\mathbf{3 9 2}$ & $36.7+/-3 \%$ & $22.7+/-11 \%$ & $36.3+/-3 \%$ \\
\hline
\end{tabular}

Looking at the values given in Table 1 it can be clearly seen that at energies of $250 \mathrm{MeV}$ and higher the detected photon contribution from the target is not anymore negligible. This conclusion is also corroborated by looking at the ratios of simulation (with and without photon component) in comparison to the measurement results depicted in Figure 5.

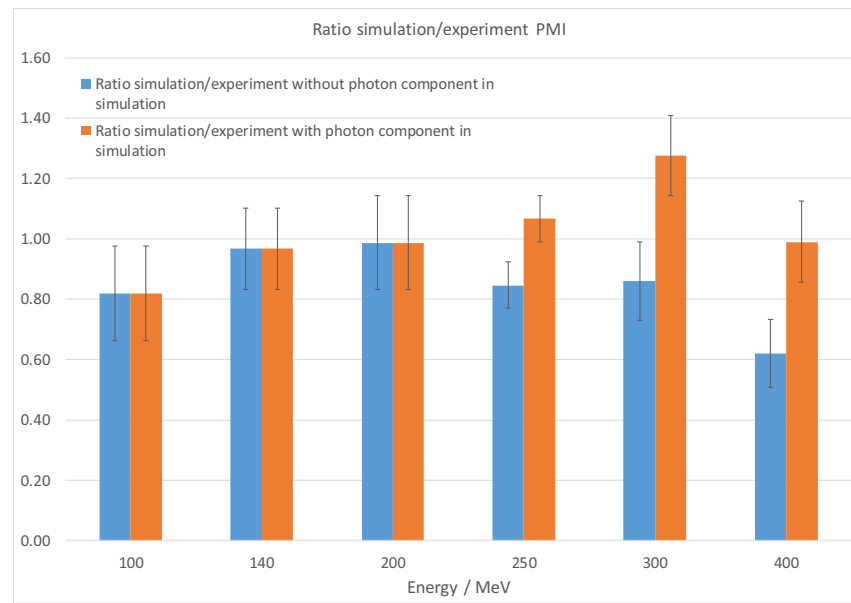

Figure 5 Comparison of the simulated PMI detector response with respect to the measurement values for quasi mono-energetic neutron energies ranging from $100 \mathrm{MeV}$ to $392 \mathrm{MeV}$. For all cases the simulation results with and without the additionally simulated photon component is shown.

As can be seen for energies of $200 \mathrm{MeV}$ and below the gamma contribution from the target reaction to the detector signal can be considered to be negligible. However, at 250 $\mathrm{MeV}$ and above a notable discrepancy can be observed if it is omitted from the simulations. At an energy range of $250-$ $300 \mathrm{MeV}$ a slight overestimation of the total signal could be suspected from the graph. This might be explained by the fact that in this energy range the approximation of the Fermi motion in the modeling of the nucleus has a greater impact than at $392 \mathrm{MeV}$ where clearly defined reaction channels for the production and decay of $\pi^{0}$ particles are present. However, considering all uncertainties the overall agreement can still be considered as quite good.

At the lowest energy of $100 \mathrm{MeV}$ a slight underestimation of the simulation can be observed in Figure 5. This might be explained by the fact that at such low energies the measurement of the proton current on the target with a Faraday cup becomes less accurate. However, this would need to be studied in detail for confirmation. In general, very good agreement of the FLUKA calculations of the PMI response to the benchmark measurements was observed. The experiment also highlighted the need to include in the simulations the complete neutron fluence spectra with the continuum as well as a thorough treatment of the photon 
component of the target reaction. While the latter can be ignored for detectors that are sensitive to neutrons only it becomes important for ionization chambers, especially at energies above $200 \mathrm{MeV}$.

\section{Conclusion}

Between 2006 and 2014 a number of measurement campaigns with quasi mono-energetic neutrons has been conducted at RCNP, Osaka University. The goal of these experiments was to verify the characterization and response simulations of PMI ionization chambers that had previously been carried out with the FLUKA Monte Carlo code. As neutrons represent a strong component in the radiation fields typically encountered at CERN's accelerators such tests in well-defined conditions represent an important complement to previous experiments that had already been carried out in mixed radiation fields at CERN.

In general very good agreement was found comparing the FLUKA simulations and the measurements. Yet, it should be emphasized that even with quasi mono-energetic neutrons the full energy spectrum has to be taken into account in the simulations to obtain reliable simulation results. In addition it was found that at proton energies of $250 \mathrm{MeV}$ and above a non-negligible gamma component originating from the target ${ }^{7} \mathrm{Li}(\mathrm{p}, \mathrm{n},)^{7} \mathrm{Be}$ reaction was observed that had to be taken into account in the simulations in order to achieve satisfying agreement. The additional photon fluence mainly originates from the creation and subsequent decay of high-energetic $\pi^{0}$ particles.

\section{Acknowledgements}

The authors would like to express their gratitude towards Alfredo Ferrari for helpful discussions and his support regarding the understanding of FLUKA's physics models.

\section{References}

1) A. Fassò, A. Ferrari, J. Ranft, and P.R. Sala, FLUKA: a multi-particle transport code, CERN Yellow Report, INFN/TC_05/11, SLAC-R-773, (2005).

2) T.T. Böhlen, F. Cerutti, M.P.W. Chin, A. Fassò, A. Ferrari, P.G. Ortega, A. Mairani, P.R. Sala, G. Smirnov and V. Vlachoudis, The FLUKA Code: Developments and Challenges for High Energy and Medical Applications, Nuclear Data Sheets 120, 211-214 (2014).

3) $\mathrm{C}$. Theis, The radiation environment in underground workplaces of the LHC, PhD thesis, TU-Graz, (2007).

4) C. Theis, D. Forkel-Wirth, M. Fuerstner, S. Mayer, Th. Otto; S. Roesler, H. Vincke, Field calibration studies for ionisation chambers in mixed high-energy radiation fields, Radiation Protection Dosimetry Advance Access published on August 1, 2007, DOI 10.1093/rpd/ncm062. Radiation Protection Dosimetry 126, pp- 299-305, (2007).

5) H. Vincke, D. Forkel-Wirth, D. Perrin, C. Theis, Simulation and measurements of the response of an air ionisation chamber exposed to a mixed high-energy radiation field, Radiation Protection Dosimetry 116 (1-4): 380-386. doi: 10.1093/rpd/nci088, (2005).
6) H. Sakai et al., Facility for the $(p, n)$ polarization transfer measurement, Nucl. Instrum. Meth. Phys. Res. A 369, 120, (1996).

7) Y. Iwamoto, M. Hagiwara, D. Satoh, H. Iwase, H. Yashima, T. Matsumoto, A. Masuda, J. Nishiyama, T. Nakamura, T. Sato, Y. Nakane, H. Nakashima, Y. Sakamoto, C. Theis, E. Feldbaumer, L. Jaegerhofer, C. Pioch, V. Mares, A. Tamii, K. Hatanaka, T. Itoga, Benchmark experiment of neutron penetration through iron and concrete shields using 138, 243 and $387 \mathrm{MeV}$ quasi-monoenergetic neutrons part-II:Measurements of neutron energy spectra, Proceedings of the SATIF 10 conference held at CERN, Switzerland, $2-4$ June (2010).

8) Y. Iwamoto, M. Hagiwara, D. Satoh, S. Araki, H. Yashima, T. Sato, A. Masuda, T. Matsumoto, N. Nakao, T. Shima, T. Kin, Y. Watanabe, H. Iwase, T. Nakamura, Characterization of high-energy quasi-monoenergetic neutron energy spectra and ambient dose equivalents of 80-389 $\mathrm{MeV} 7 \mathrm{Li}(p, n)$ reactions using a time-of-flight method, Nuclear Instrument and Methods A 804, 50 (2015).

9) A. Ferrari and P.R. Sala, The physics of high energy reactions, Proceedings of Workshop on nuclear reaction data and nuclear reactors physics, design and safety, Trieste, (1996).

10) S. G. Mashnik, M. B. Chadwick, H. G. Hughes, R. C. Little, R. E. MacFarlane, L. S. Waters, P.G. Young, ${ }^{7} L i(p, n)$ nuclear data library for incident proton energies to $150 \mathrm{MeV}$, Proceedings 2000 ANS/ENS International Meeting, Nuclear Applications of Accelerator Technology (AccApp00), (2000).

11) C. Urscheler, Radiological studies for the luminosity upgrade of the CMS detector, PhD thesis at the ETH Zurich, Diss. ETH No. 20578, (2012). 